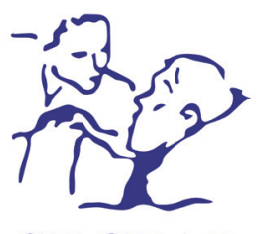

Medicina Paliativa

www.medicinapaliativa.es

ORIGINAL

\title{
Supervivencia tras delirium en pacientes oncológicos avanzados tratados por profesionales específicos de cuidados paliativos en ámbito hospitalario: ¿hay alguna diferencia?
}

\author{
Claudio Calvo Espinós ${ }^{1 *}$, Beatriz Galañena Jamar ${ }^{2}$, Marcos Lama Gay ${ }^{3}$ \\ y Estefanía Ruiz de Gaona Lana ${ }^{4}$
}

${ }^{1}$ Servicio de Cuidados Paliativos, Fundación Rioja Salud. La Rioja, España. ${ }^{2}$ Atención Primaria, Centro de Salud San Juan. Pamplona, Navarra, España. ${ }^{3}$ Atención Primaria, Centro de Salud Isaba. Navarra, España. ${ }^{4}$ Servicio de Hematología y Hemoterapia, Fundación Hospital Calahorra. La Rioja, España

Recibido el 17 de enero de 2020

Aceptado el 12 de abril de 2020

\author{
PALABRAS CLAVE \\ Delirium, cuidados \\ paliativos, pronóstico.
}

\begin{abstract}
Resumen
Introducción: El delirium es un factor pronóstico negativo en población geriátrica y de enfermedad avanzada. No hay trabajos que focalicen en la población atendida por equipos específicos de cuidados paliativos.

Objetivo: Analizar la supervivencia de los pacientes que debutan con un cuadro de delirium durante su estancia en una Unidad de Hospitalización de Cuidados Paliativos.

Metodología: Estudio descriptivo retrospectivo de supervivencia. Se incluyeron pacientes ingresados en la UCP del HSJD de Pamplona durante el semestre abril-septiembre 2012. Variables: datos demográficos, tipo de neoplasia, deterioro cognitivo, ECOG y supervivencia desde el ingreso. Se registró la presencia de delirium según criterios DSM IV al ingreso y durante el ingreso. De este último subgrupo se registraron las variables: factores precipitantes-contribuyentes, subtipo de delirium, reversibilidad del cuadro y supervivencia desde el diagnóstico. Se calcularon funciones de supervivencia mediante el método de Kaplan-Meier. Se comparó supervivencia respecto a presentar o no delirium mediante la prueba de mantel-haenszel (log-rank).

Resultados: Prevalencia de delirium del 50 \% (26\% al ingreso) con incidencia del $25 \%$. Mediana supervivencia: 11 días (IC 7,54-14,45) en los pacientes con delirium y de 22 días (IC 11,41-32,58) en el resto. En los que debutaron durante el ingreso, la mediana de supervivencia fue 16 días y 5 desde el diagnóstico de delirium. Existen diferencias estadísticamente significativas entre la supervivencia del grupo que ingresa con delirium frente a los que no lo presentan en ningún momento, así como la suma del total de pacientes con delirium. No hubo diferencias significativas entre los que debutan durante el ingreso y los que no presentan delirium.
\end{abstract}

\footnotetext{
*Autor para correspondencia:

Claudio Calvo Espinós

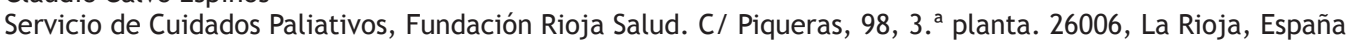

Correo electrónico: clacales75@yahoo.es
}

DOI: $10.20986 /$ medpal.2020.1136/2020

1134-248X/ ( 2020 Sociedad Española de Cuidados Paliativos. Publicado por Inspira Network. Todos los derechos reservados. 
Conclusiones: El delirium ha demostrado ser un factor pronóstico a nivel global. Sin embargo, en poblaciones con mayor fragilidad y en seguimiento continuo por personal entrenado en el manejo del mismo, su influencia diferencial sobre la supervivencia no ha quedado tan claramente establecida.

\section{KEYWORDS}

Delirium, palliative care, prognosis.

Calvo Espinós C, Galañena Jamar B, Lama Gay M, Ruiz de Gaona Lana E. Supervivencia tras delirium en pacientes oncológicos avanzados tratados por profesionales específicos de cuidados paliativos en ámbito hospitalario: ¿hay alguna diferencia? Med Paliat. 2020;27(2):98-105

\section{Introducción}

El delirium es el estado confusional agudo que resulta de una disfunción orgánica cerebral difusa y que se caracteriza por alteraciones en el nivel de conciencia y atención, asociándose alteraciones cognitivas y de la percepción ${ }^{1}$. El delirium provoca un impacto en la capacidad de comunicación del paciente, en la capacidad de tomar decisiones, en la capacidad funcional y en la calidad de vida ${ }^{2}$, y no solo en los pacientes sino también en sus familiares, e incluso en los profesionales que les atienden ${ }^{3}$. Los pacientes con enfermedad oncológica avanzada presentan un gran riesgo de delirium a lo largo de su evolución. Supone una de las primeras causas de ingreso hospitalario en una Unidad de Paliativos ${ }^{1,4}$, además de un factor que incrementa el coste de atención sanitaria ${ }^{5}$. Respecto a su manejo, precisa de profesionales entrenados en su evaluación y tratamiento ${ }^{6-8}$, ya que es un claro factor de confusión en la expresión sintomática de los pacientes 9 .

Su incidencia en medicina paliativa oscila entre un 28 y un $88 \%$, dependiendo de la etapa-estadio de la enferme$\operatorname{dad}^{7,8,10-12}$. Al ingreso en una unidad de cuidados paliativos su frecuencia se halla entre el 26 y el $44 \%$ 6-8,11-14. Es más frecuente en estadios muy avanzados, pudiendo afectar en situación de últimos días hasta al 83-90\% de los enfer$\operatorname{mos}^{4,13,14}$. En resumen, la incidencia de delirium aumenta con el deterioro de la condición general del enfermo y es mayor cuanto más terminal es la situación del paciente ${ }^{10}$.

El delirium es un marcador independiente de mayor riesgo de mortalidad en pacientes mayores hospitalizados, tanto en enfermos en los que no existe demencia previa ${ }^{4}$ como en los que sí la padecen ${ }^{15}$. En otros estudios en población geriá- 
trica, la mortalidad asociada a delirium no es significativa cuando se ajusta a la severidad de la comorbilidad ${ }^{16,17}$. La reversibilidad es el principal foco de estudio recomendado en fases incipientes ${ }^{18,19}$. En enfermos con cáncer avanzado se ha establecido como factor independiente de mal pronóstico respecto a la supervivencia, demostrando que su impacto es especialmente importante en pacientes con una relativo mejor pronóstico 20 .

Hay pocos trabajos que analicen la importancia pronóstica del delirium en la población atendida por equipos específicos de cuidados paliativos ${ }^{11,21}$. Las medias y medianas de supervivencia en estos casos, y en los distintos entornos de atención, presentes en la bibliografía, muestran grandes fluctuaciones $^{11}$. Esto seguramente es debido a la complejidad para filiar bien los factores asociados al delirium (predisponentes y desencadenantes) ${ }^{22}$, así como por la misma dificultad de realizar un buen diagnóstico. Además, no está bien definido en la bibliografía el impacto sobre la incidencia y la reversibilidad del delirium que la formación específica paliativa puede tener ${ }^{23}$. Como objetivo primario de nuestro estudio, nos preguntamos qué supervivencia tienen los pacientes que debutan con delirium durante el ingreso en nuestra unidad de cuidados paliativos. Secundariamente, nos proponemos analizar la diferencia entre los que lo presentan al ingreso, debutan posteriormente, y los que no lo desarrollan nunca durante su evolución.

\section{Metodología}

Se realizó un estudio retrospectivo descriptivo de supervivencia. Se incluyeron a todos los pacientes mayores de 18 años con diagnóstico de enfermedad oncológica avanzada, en los que se ha desestimado cualquier tratamiento específico con intención curativa, que ingresaron en la Unidad de Hospitalización de Cuidados Paliativos del Hospital San Juan de Dios (HSJD) de Pamplona en el semestre (abril-septiembre) de 2012. Quedaron, por tanto, excluidos los pacientes menores de 18 años, pacientes con enfermedad no oncológica y pacientes con enfermedad oncológica que estaban recibiendo tratamiento específico con intención curativa.

La recogida de datos se realizó revisando la historia clínica informatizada de los pacientes ingresados durante ese periodo, previa autorización por parte del comité de ética asistencial del hospital. En estos pacientes se recogieron las siguientes variables: edad, sexo, grado de funcionalidad al ingreso a través del Eastern Cooperative Oncology Group (ECOG), presencia de deterioro cognitivo previo, supervivencia desde el ingreso y tipo de neoplasia. A este respecto, agrupamos la neoplasia primaria en: pulmón (broncopulmonar y pleural), aparato digestivo (esofágica, gástrica, colon, hepática y pancreática), genitourinario (renal, vesical, próstata, útero, ovario), mama, sistema nervioso central (SNC) y otras (melanoma, tiroides, primario desconocido).

Respecto a la presencia o no de delirium, se tuvo en cuenta que los datos clínicos registrados en la historia clínica concordasen con los criterios diagnósticos DSM IV (Manual Diagnóstico y Estadístico de las Enfermedades Mentales, cuarta edición) para delirium, que son:

A. Alteración de la capacidad para centrar, mantener o dirigir la atención.
B. La alteración se presenta en un corto periodo de tiempo (habitualmente en horas o días) y tiende a fluctuar a lo largo del día.

C. Cambio en las funciones cognoscitivas (como deterioro de la memoria, desorientación, alteración del lenguaje) o presencia de una alteración perceptiva que no se explica por una demanda previa o en desarrollo.

D. Alteración del nivel de conciencia (desde hiperalerta hasta hipoactivo).

Para codificarlo como "delirium" en nuestro estudio, se precisaba la presencia de: un registro de "delirium" o "síndrome confusional agudo" o "cuadro confusional"; o los dos primeros criterios del manual DSM IV $(A, B)$ junto con, al menos, uno de los dos siguientes (C,D). No se registró como "delirium" a los pacientes en cuyas historias constan términos imprecisos tales como "fallo cognitivo, inquietud, agitación terminal o alucinaciones" con la intención de evitar en lo posible falsos positivos. Tampoco se incluyeron en el grupo delirium aquellos pacientes en cuyas historias constan términos sugestivos de delirium en las 24 horas antes del fallecimiento. En los últimos días, casi todos los pacientes sufren una disfunción cerebral difusa. Se ha discutido si el delirium y la agitación terminal son entidades distintas, o si son simplemente el mismo problema en distintos momentos de la historia natural de la enfermedad de un paciente. No es clara la respuesta a esta cuestión ${ }^{4}$. Se decidió no incluir a estos pacientes para poner el foco en los delirium con más opciones de reversibilidad, y para evitar falsos positivos.

En el grupo de pacientes seleccionados bajo estos criterios se recogieron las variables "presencia de delirium al ingreso" y "debut de delirium durante el ingreso". El objetivo de ello fue analizar ambos grupos de forma independiente, ya que la presencia de delirium al ingreso puede dificultar el proceso diagnóstico etiológico, mientras que el que debuta durante el seguimiento puede ser más categorizado $\mathrm{y}$, por tanto, tratado.

En el subgrupo “debut de delirium durante el ingreso" se recogieron, además, las siguientes variables: supervivencia desde el diagnóstico de delirium, reversibilidad (considerando como tal cuando ya no cumplía ninguno de los criterios diagnósticos de DSM IV en valoraciones en 3 días sucesivos); subtipo de delirium (hiperactivo, hipoactivo o mixto), y factores contribuyentes-precipitantes. Se consideraron como factores contribuyentes-precipitantes aquellos que cumpliesen las siguientes condiciones: a) que fuera un factor ya descrito como causa de delirium; b) que hubiera relación temporal con la aparición del cuadro; y c) que quede reflejado en la historia clínica como probable factor etiológico. Estos factores se agruparon de la siguiente manera: enfermedad orgánica cerebral que incluye tumores primarios o metástasis sobre SNC en progresión; carcinomatosis meníngea; enfermedad cerebrovascular); y causas tóxico-metabólicas (empeoramiento función renal; anemia; insuficiencia de órgano; alteraciones metabólicas como hipercalcemia; proceso infeccioso intercurrente; introducción o modificación de dosis de opioides y/o psicofármacos).

El análisis estadístico fue descriptivo de las variables sociodemográficas y clínicas. Además, se calcularon funciones de supervivencia mediante el método de Kaplan-Meier. Se comparó la supervivencia respecto a presentar o no delirium mediante la prueba de Mantel-Haenszel (log-rank). 


\section{Resultados}

Durante el semestre abril-septiembre de 2012 ingresaron en la unidad de cuidados paliativos 200 pacientes. Se incluyeron en el estudio 192 pacientes, quedando excluidos 8 pacientes; tres de ellos por padecer enfermedades avanzadas no oncológicas (esclerosis lateral amiotrófica [ELA], Enfermedad de Creutzfeldt-Jakob) y cinco quedan excluidos por estar recibiendo tratamiento específico con intención curativa (Figura 1).

El perfil de paciente fue un varón en torno a 70 años con una localización primaria en el aparato digestivo (Tabla I). El estatus funcional más frecuente fue ECOG $=3-4$, con una presencia de deterioro cognitivo previo de $24 \%$.

El perfil del grupo que pacientes que debutaron con delirium durante el ingreso se muestra en la Tabla II. La prevalencia de delirium en nuestra muestra fue de casi del $50 \%$, con una incidencia del $25 \%$ durante el ingreso y del $26 \%$ en el momento del ingreso (Tabla III). El cuadro de delirium fue

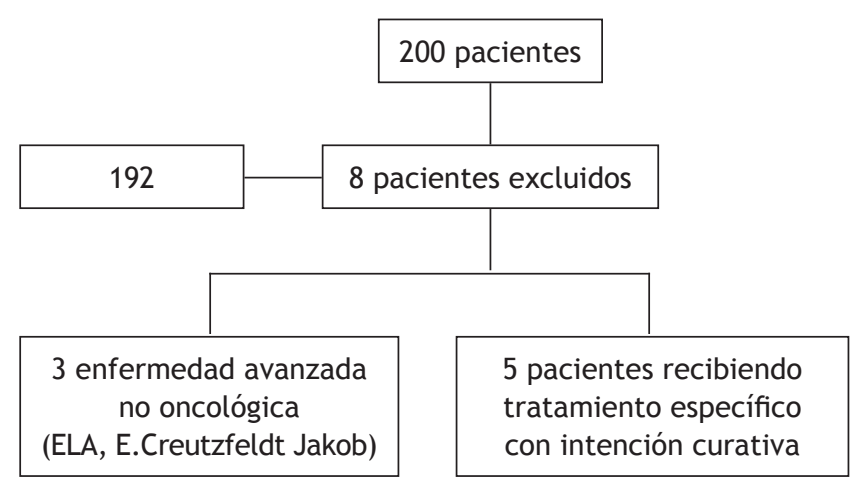

Figura 1. Diagrama de flujo.

Tabla I. Variables sociodemográficas y clínicas

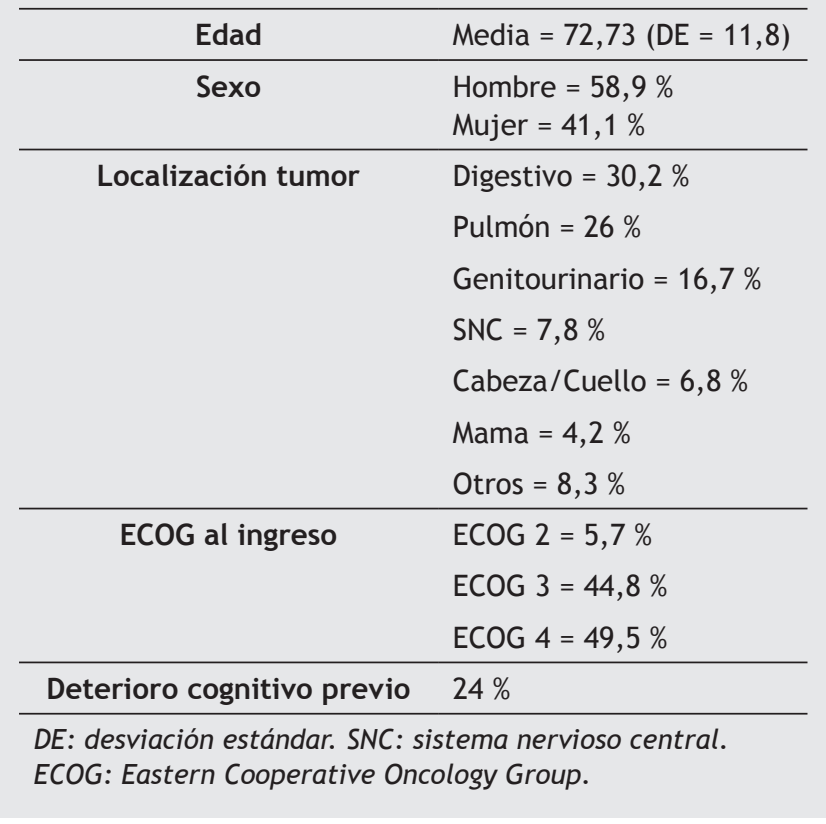

Tabla II. Perfil del paciente con delirium durante el ingreso $(n=48)$

\begin{tabular}{cl}
\hline Edad & Media $=74,81(\mathrm{DE}=10,2)$ \\
\hline \multirow{2}{*}{ Sexo } & Hombre $=67 \%$ \\
& Mujer $=33 \%$ \\
\hline & Digestivo $=31 \%$ \\
& Pulmón $=23 \%$ \\
& Genitourinario $=14,5 \%$ \\
Localización tumor & SNC $=10,4 \%$ \\
& Cabeza $/$ Cuello $=6,2 \%$ \\
& Mama $=2 \%$ \\
& Otros $=12,5 \%$ \\
\hline & ECOG $2=4,1 \%$ \\
ECOG al ingreso & ECOG 3 $=60,41 \%$ \\
& ECOG $4=35,4 \%$ \\
\hline Deterioro cognitivo previo & $20,8 \%$ \\
\hline DE: desviación estándar. SNC: sistema nervioso central. \\
ECOG: Easten Cooperative Oncology Group.
\end{tabular}

Tabla III. Resultados de delirium

\begin{tabular}{cl}
\hline Delirium (total) & $49 \%$ \\
\hline Delirium al ingreso & $26 \%$ \\
\hline $\begin{array}{c}\text { Delirium durante } \\
\text { el ingreso }\end{array}$ & $25 \%$ \\
\hline Reversibilidad & $16,2 \%$ \\
\hline Tipo & Mixto $=44,3 \%$ \\
\hline & Hiperactivo $=43,3 \%$ \\
\hline Causa probable & Hipoactivo $=12,4 \%$ \\
\hline & Desconocida $=58,1 \%$ \\
\hline & Toxico-metabólica $=33,3 \%$ \\
\hline & Enfermedad $=8,6 \%$
\end{tabular}

reversible solo en un $16 \%$ de las veces, siendo la hiperactiva y la mixta la presentación más frecuente (en torno al $44 \%$ ambas). La etiología más frecuente fue la desconocida (58\%).

La supervivencia en el global de la muestra fue en torno a 60 días de media, con una mediana de 14 días (Tabla IV y Figura 2). Dividiendo la muestra según la presencia de delirium, ya sea al ingreso o durante el mismo, las supervivencias van desde una mediana de 22 días en los que no lo presentan en ningún momento, a 5 días en los que ingresan ya con ese diagnóstico. La supervivencia desde el día de diagnóstico en los que debutan durante el ingreso es de 8 días (16 desde el día de ingreso), con una mediana de 11 en el grupo de todos los pacientes que presentan un delirium (ya sea al ingreso o durante él) (Figura 3). Las probabilidades de supervivencia en días en los tres grupos antes descritos se reflejan en la Tabla V. Se observa una tendencia a una 
Tabla IV. Supervivencias

\begin{tabular}{lcccc}
\hline & Media (días) & IC & Mediana (días) & IC \\
\hline Sv. global & 59,34 & $43,97-74,71$ & 14 & $10,91-17,09$ \\
\hline Grupo con delirium durante el ingreso (1) & & & & $3,87-12,13$ \\
- Sv. desde dx delirum & 23,62 & $11,2-36,05$ & 13 & $12,76-19,23$ \\
- Sv. desde el ingreso & 45,34 & $24,81-65,87$ & 5 & $3,27-6,73$ \\
\hline Sv. delirium al ingreso (2) & 26,14 & $9,39-42,89$ & 11 & $7,54-14,45$ \\
\hline Sv. total delirium (1+ 2) & 36,25 & $21,95-50,55$ & 22 & $11,41-32,58$
\end{tabular}

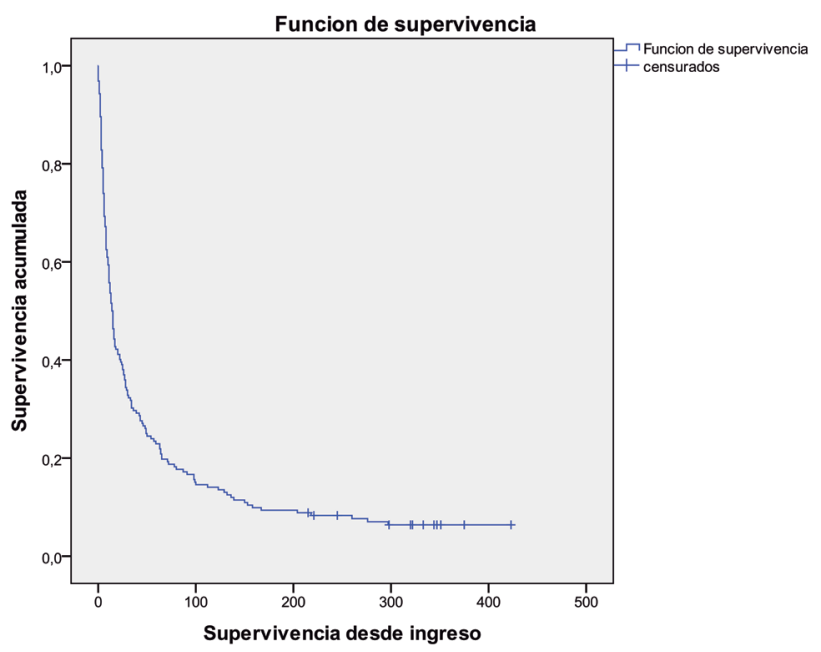

Figura 2. Curva Kaplan-Meier de supervivencia global.

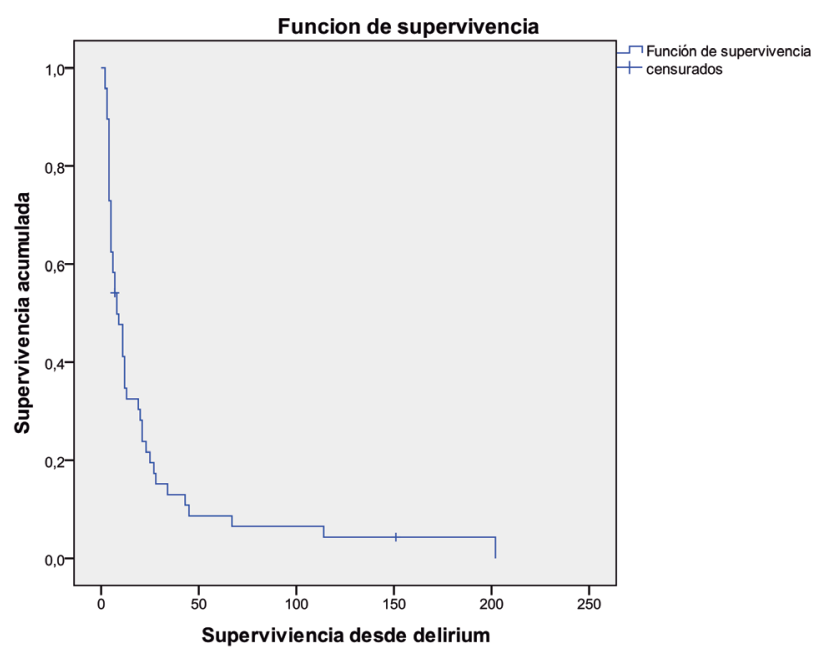

Figura 3. Curva Kaplan-Meier de supervivencia desde debut delirium.
Tabla V. Probabilidad de supervivencia en días

\begin{tabular}{ccccc}
\hline & $\mathbf{7}$ días & $\mathbf{1 4}$ días & $\mathbf{2 1}$ días & $\mathbf{2 8}$ días \\
\hline No delirium & $71 \%$ & $41 \%$ & $21 \%$ & $10 \%$ \\
\hline $\begin{array}{c}\text { Delirium al } \\
\text { ingreso }\end{array}$ & $44 \%$ & $11 \%$ & $2,5 \%$ & $0,5 \%$ \\
\hline $\begin{array}{c}\text { Desde el } \\
\text { diagnóstico de } \\
\text { delirium }\end{array}$ & $60 \%$ & $20,4 \%$ & $5,9 \%$ & $1 \%$ \\
& & & &
\end{tabular}

menor supervivencia desde el debut de delirium, comprobada claramente en el grupo de pacientes que ya ingresa con el diagnóstico.

Existen diferencias estadísticamente significativas entre la supervivencia del grupo que ingresa ya con delirium frente a los que no lo presentan en ningún momento (log Rank $\mathrm{p}=0,000)$, así como la suma del total de pacientes con delirium (al ingreso y durante el ingreso) frente a los que no lo presentan (log Rank $p=0,005)$ (Figura 4). En cambio, no se observan diferencias significativas entre los que debutan durante el ingreso y los que no presentan delirium (log Rank $\mathrm{p}=0,35)$ (Figura 5).

\section{Discusión}

Este trabajo analiza la supervivencia tras un episodio de delirium en pacientes ingresados en una unidad de cuidados paliativos. Predecir la supervivencia es necesario para una correcta actuación clínica y una buena organización, además de por razones éticas, para ayudar a evitar daños, incomodidad y terapias inapropiadas en pacientes vulnerables $^{24}$. Conociendo la supervivencia de los pacientes que desarrollan delirium en nuestra unidad, podremos determinar con mayor precisión el pronóstico, y con ello ayudaremos a los pacientes y sus familias a tomar mejores decisiones. Nuestros resultados confirman la tendencia ya presente en la bibliografía del impacto negativo en la supervivencia en 


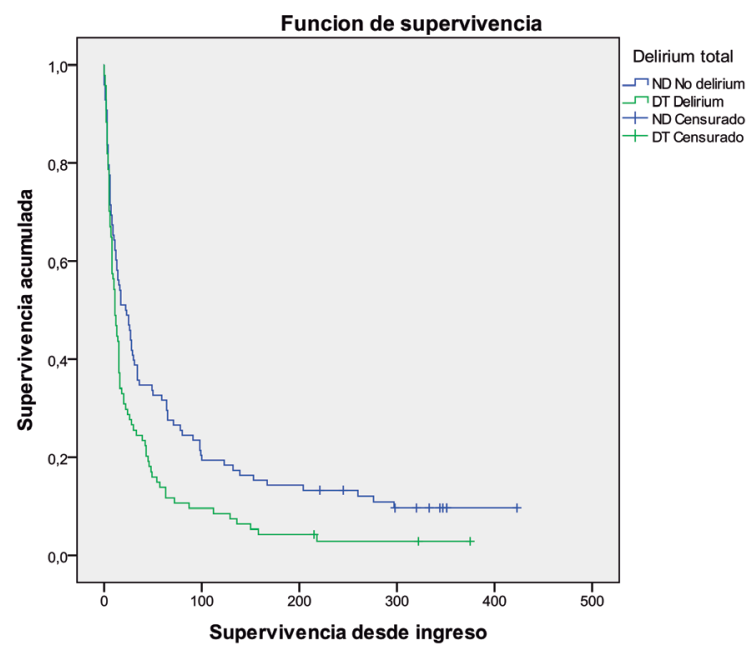

Figura 4. Curva Kaplan-Meier de supervivencia de delirium total vs. no delirium.

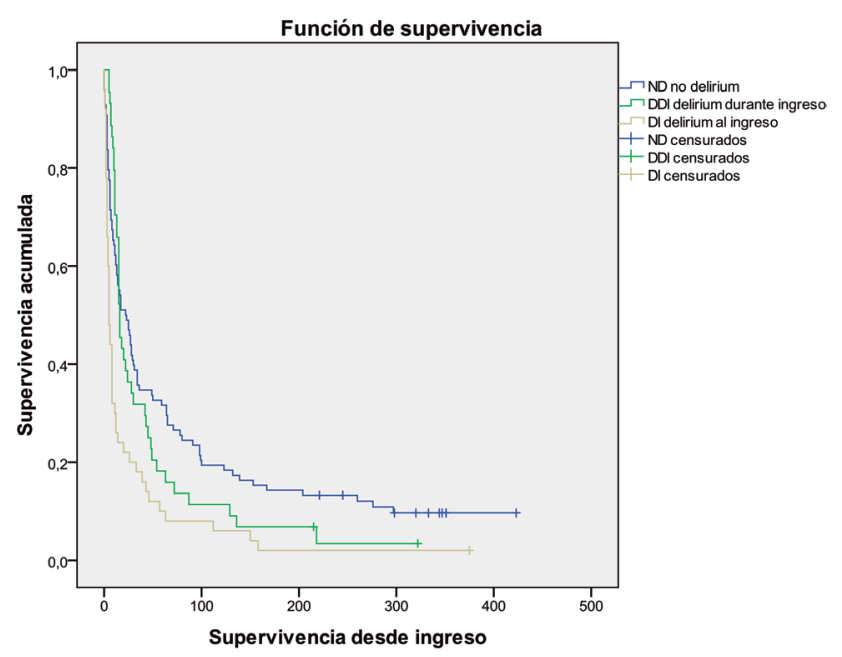

Figura 5. Curva Kaplan-Meier de supervivencia en los 3 grupos.

los pacientes con delirium. Sin embargo, siembran dudas respecto a aquellos que debutan durante el seguimiento de un equipo específico de cuidados paliativos en régimen de ingreso, presentando un menor impacto y no significativo. Creemos que esto es relevante para conocer mejor a la población atendida en nuestras unidades.

La población que ingresa en unidades de cuidados paliativos muestra unos rangos de edad, sexo y localización primaria muy similares a los de nuestro trabajo. El perfil de paciente suele ser de una edad en torno a los 70 años $2,13,20,25$, con más frecuencia de varones ${ }^{13,2,26}$. La neoplasia primaria se localiza más frecuentemente en aparato digestivo y pulmón ${ }^{1,20,25-26}$. Nuestro trabajo confirma estos datos (varón de 72 años con un cáncer gastrointestinal o pulmonar como localizaciones más frecuentes). Esto muestra la homoge- neidad de nuestra unidad respecto a la reflejada en otros estudios $^{1,13,20,25}$.

El estatus funcional más frecuente en el momento del ingreso es ECOG 4, también similar a estudios previos ${ }^{20}$. Esto indica el bajo estatus funcional de los pacientes que ingresan en nuestra unidad. Es conocida la importancia pronóstica del estatus funcional ${ }^{24}$, lo cual, a la vista de la supervivencia mostrada en nuestro trabajo, demuestra la frágil situación en la que ingresan nuestros pacientes. Este es un factor muy a tener en cuenta, en nuestra opinión, a la hora de comparar nuestros resultados con los mostrados en la literatura.

El delirium puede presentarse en pacientes sin patología cerebral previa, pero es más frecuente en los que presentan una enfermedad de base como la demencia ${ }^{4}$. Es conocida la mayor vulnerabilidad en población geriátrica ${ }^{27}$. También en el enfermo de cáncer, la demencia o cualquier otro déficit cognitivo previo aumentan el riesgo de padecer delirium ${ }^{28}$. Los trabajos consultados en el área de cuidados paliativos frecuentemente omiten la situación cognitiva basal. Otros estudios, más en el ámbito de la geriatría, presentan cifras en torno al $20 \%$ de deterioro cognitivo previo (19-26\%) $)^{17,29}$, muy similares a los mostrados en nuestro estudio. No es objeto de este estudio valorar los factores de riesgo precipitantes, por lo que no hemos analizado los datos referentes a este subgrupo.

Dentro del grupo de pacientes que desarrollaron delirium durante el ingreso, se percibe un discreto aumento de la edad media (75 años). Este dato concuerda con otros estudios que analizan los factores de riesgo, siendo la edad avanzada uno de los factores que aumenta el riesgo de desarrollar delirium ${ }^{4,27-30}$. Además se percibe en este subgrupo un aumento de localización primaria en el SNC. Este dato es coherente con la fisiopatología del delirium. Asimismo, se observa un mayor porcentaje de hombres respecto al total de la muestra, dato no confirmado por estudios recientes ${ }^{2}$. Es llamativa la escasa tasa de deterioro cognitivo previo en esta población, siendo este un claro predisponerte o desencadenante de delirium, como ya se ha comentado.

Un $26 \%$ de los pacientes que ingresan cumplen criterios diagnósticos de delirium, que es consistente con los resultados encontrados en otros estudios de prevalencia al ingreso $(13,3-45 \%)^{2}$. Datos de una reciente revisión sistemática sobre prevalencia e incidencia de delirium, se estima esta última en un rango entre 3-45\%, siendo en nuestra muestra $25 \%{ }^{12}$. Otros estudios miden la prevalencia de delirium en las semanas-horas antes de la muerte presentando unas cifras de 58,8-88 \%. Nuestros datos no concuerdan con estos últimos resultados, probablemente por la decisión, ya detallada en la metodología, de haber excluido de la muestra de delirium a los pacientes en cuyas historias constan términos imprecisos como "agitación terminal”, "confusión” o "alucinaciones", así como términos sugestivos de delirium en las 24 horas antes del fallecimiento. Somos conscientes del posible aumento de falsos negativos que esta opción ha podido provocar. Sin embargo, estamos convencidos de que la decisión contraria de incluirlos en la muestra total, más habitual en el resto de estudios consultados, puede resultar un factor de confusión no controlado respecto al peso pronóstico del delirium.

En nuestra muestra el subtipo más prevalente fue el mixto. Estos datos concuerdan con estudios previos ${ }^{1}$, pero no con algún trabajo más reciente (Hosie y cols. $)^{2}$. Esta discor- 
dancia nos parece coherente con la dificultad de diagnóstico de la presentación hipoactiva de delirium. Otro condicionante es la metodología retrospectiva de nuestro trabajo, que depende de la calidad de los registros analizados.

Leonard y cols. ${ }^{13}$ analizan en un grupo de 121 pacientes diagnosticados de delirium la reversibilidad y los factores relacionados con la misma. El 33,27 \% de los casos en este trabajo fue reversible. Otros trabajos muestran que aproximadamente el $50 \%$ de los episodios de delirium pueden ser reversibles $8,14,18,31$. Trabajos más recientes confirman estas cifras $^{22}$. Otros estudios, en cambio, muestran tasas inferiores en torno al 7,14\%1. En nuestro caso, el $16,2 \%$ de los episodios fueron reversibles, sin poder determinar con los datos registrados los factores desencadenantes-precipitantes y su relación con la reversibilidad del cuadro.

Existe cierta base en nuestro ámbito, más claramente establecido en geriatría, acerca del peso pronóstico del delirium ${ }^{20}$. Sin embargo, no existen muchos trabajos que analicen la supervivencia, al menos en el área de cuidados paliativos. Los pocos que hay muestran claras diferencias metodológicas respecto al nuestro. En concreto, en lo referente a los pacientes incluidos dentro de los que debutaron con delirium, hay trabajos en los que se incluyen en este grupo a todo paciente que ingresa o debuta con cuadro confusional 1 1,20,25. Además, existe debate, como ya se ha detallado anteriormente, en torno a si incluir o no a aquellos pacientes que inician delirium en las últimas horas de vida. Por ejemplo, Leonard y cols. ${ }^{13}$ excluyen de la muestra de delirium a aquellos pacientes cuyo equipo sanitario considera de elevada probabilidad de fallecer en las próximas horas. En cambio, Lawlor y cols. ${ }^{21}$ clasifican e incluyen en su estudio a este subgrupo bajo el concepto de "delirium terminal". A la hora de plantear nuestro estudio, consideramos que el objetivo sobre el que poner nuestro foco era aquel delirium no directamente relacionado con la situación de últimos días u horas. En la llamada situación de agonía, casi todos los pacientes sufren una disfunción cerebral difusa. Se discute si el delirium y la agitación terminal son entidades distintas, o si son simplemente el mismo problema en momentos distintos de la historia natural de la enfermedad. No es clara la respuesta a esta cuestión ${ }^{4}$. Además, estudios clásicos en torno a la situación de agonía (Ellershaw y cols.) definen la disfunción cognitiva como uno de los más claros signos asociados a la cercanía del final. Junto a esto, las dificultades para filiar con claridad el inicio del cuadro en aquellos pacientes que ingresaron ya bajo el diagnóstico de delirium, nos movió a registrar ese grupo de forma independiente, con vistas a detectar diferencias entre ingresar ya con un cuadro de delirium o desarrollarlo durante el ingreso. Esta característica de nuestro estudio hace difícil la comparación con otros trabajos en este ámbito, debido a que la mayoría incluyen tanto a la muestra de pacientes global (los que ingresan y los que debutan) como a los que presentan disfunción cognitiva en las horas previas a la muerte.

Caraceni y cols. ${ }^{20}$ analizaron, en una población similar a la nuestra, aunque con un menor número de pacientes incluidos, la supervivencia de los pacientes que presentaron delirium (21 días) frente a los que no presentaron delirium (39 días), mostrando una diferencia estadísticamente significativa entre ambas. En nuestra muestra, se observa una tendencia a una menor supervivencia desde el debut de delirium, comprobada claramente en el grupo de pacientes que ya ingresa con delirium. Existen diferencias estadísticamente significativas entre la supervivencia del grupo que ingresa ya con delirium frente a los que no lo presentan en ningún momento. Asimismo, la suma del total de pacientes con delirium frente a los que no lo presentan arroja datos similares. Estos datos concuerdan con los obtenidos por Caraceni y cols. ${ }^{20}$. Sin embargo, en el subgrupo que debuta con delirium durante el ingreso no se observan diferencias significativas de supervivencia frente al grupo de no-delirium. Caraceni y cols. afirman en su estudio que el deterioro de las funciones superiores es un factor pronóstico independiente en pacientes con cáncer avanzado, demostrando que el impacto es particularmente importante en los pacientes con un mejor pronóstico. Nuestro trabajo confirma lo anterior, aunque creemos que arroja luz sobre esa población de nuestras unidades de hospitalización. En nuestro caso, se observa claramente que el perfil del paciente que es remitido a nuestra unidad presenta un peor estatus funcional desde el ingreso (ECOG 3-4), confirmando las peores expectativas con una mediana de supervivencia de 14 días frente a 33 días en los pacientes del trabajo anterior ${ }^{20}$.

En la misma línea, O'Keeffe y cols. ${ }^{17}$, desde el ámbito de la geriatría, obtienen resultados similares a los nuestros, afirmando que la mortalidad asociada a delirium no es significativa cuando se ajusta según la edad, la comorbilidad, la severidad de la enfermedad o la demencia. Asimismo, Leonard y cols. ${ }^{13}$ consideran que la supervivencia en pacientes terminales que desarrollan delirium, se relaciona especialmente con la edad, el grado de severidad del deterioro cognitivo y el fallo de órgano. Estos datos avalan nuestros resultados, ya que, como hemos manifestado previamente, la población de nuestro estudio se enmarca en estos criterios, a tenor de la mediana de supervivencia global obtenida $y$ del estatus funcional.

El diseño del estudio lleva inherente una serie de limitaciones al ser un estudio retrospectivo. Al escorarnos para evitar falsos positivos, es posible que exista un aumento en la probabilidad de falsos negativos y las consecuencias que esto supone. Podemos aportar poca información respecto a la etiología, factores predisponentes-desencadenantes y subtipo de delirium debido fundamentalmente a la calidad de los registros en esas áreas. Consideramos que hubiera aportado información tener un grupo control de pacientes desde el diagnóstico de delirium durante el ingreso para así hacer una comparativa directa de supervivencia desde esa fecha.

Como conclusión, creemos que nuestro estudio aporta luz sobre el complejo ámbito del delirium en el área de los cuidados paliativos. Consideramos que los datos, contrastados por la bibliografía muestran que el delirium es un factor con peso pronóstico especialmente en aquellos pacientes con una mejor expectativa de supervivencia que en aquellos pacientes más frecuentemente ingresados en nuestras unidades de hospitalización. En esta población, con una supervivencia en su gran mayoría inferior a las tres semanas, y con unos profesionales bien entrenados en la evaluación y el tratamiento de esta patología, el peso pronóstico de debutar con un cuadro de delirium se ha demostrado menor. Son necesarios más estudios, especialmente de metodología prospectiva, para confirmar estos indicios. 


\section{Bibliografía}

1. Calsina Berna A, Garzón Rodriguez C, Porta Sales J. Delirium en pacientes oncológicos avanzados en una unidad de hospitalización de cuidados paliativos. Med Paliat. 2009;16;286-90.

2. Hosie A, Davidson P, Agar M, Sanderson C, Phillips J. Delirium prevalence, incidence, and implications for screening in specialist palliative care inpatient settings: A systematic review. Palliat Med. 2013;27:486-98.

3. Schmitt EM, Gallagher J, Albuquerque A, Tabloski P, Lee HJ, Gleason L, et al. Perspectives on the Delirium Experience and Its Burden: Common Themes Among Older Patients, Their Family Caregivers, and Nurses. Gerontologist. 2019;59:327-37.

4. Centeno C, Vara F, Pérez P, Sanz A, Bruera E. Presentación clínica e identificación del delirium en el cáncer avanzado. Med Paliat. 2003;10:24-35.

5. Pezzullo L, Streatfeild J, Hickson J, Teodorczuk A, Agar MR, Caplan GA. Economic impact of delirium in Australia: a cost of illness study. BMJ Open. 2019;9:e027514.

6. Boland JW, Kabir M, Bush SH, Spiller JA, Johnson MJ, Agar M, et al. Delirium management by palliative medicine specialists: a survey from the association for palliative medicine of Great Britain and Ireland. BMJ Support Palliat Care. 2019. pii: bmjspcare-2018-001586. [Epub ahead of print]

7. Bush SH, Lawlor PG, Ryan K, Centeno C, Lucchesi M, Kanji S, et al. Delirium in adult cancer patients: ESMO Clinical Practice Guidelines. Ann Oncol. 2018;29:iv143-iv165.

8. Bush SH, Tierney S, Lawlor PG. Clinical Assessment and Management of Delirium in the Palliative Care Setting. Drugs. 2017;77:1623-43.

9. Mercadante S, Adile C, Ferrera P, Cortegiani A, Casuccio A. Symptom Expression in Patients with Advanced Cancer Admitted to an Acute Supportive/Palliative Care Unit With and Without Delirium. Oncologist. 2019;24:e358-e364.

10. LeGrand SB. Delirium in Palliative Medicine: A review. J Pain Symptom Manage. 2012;44:583-94.

11. Pallotti MC, López-Fidalgo J, Biasco G, Celin D, Centeno C, Paragona M, et al. Delirium Rates in Advanced Cancer Patients Admitted to Different Palliative Care Settings: Does It Make the Difference? J Palliat Med. 2019. [Epub ahead of print].

12. Watt CL, Momoli F, Ansari MT, Sikora L, Bush SH, Hosie A, et al. The incidence and prevalence of delirium across palliative care settings: A systematic review. Palliat Med. 2019;33:865-77.

13. Leonard M, Raju B, Conroy M, Donnelly S, Trzepacz PT, Saunders $\mathrm{J}$, et al. Reversibility of delirium in terminally ill patients and predictors of mortality. Palliat Med. 2008;22:848-54.

14. Bush SH, Bruera E. The Assessment and Management of Delirium in Cancer Patients. Oncologist. 2009;14:1039-49.

15. Morandi A, Di Santo SG, Zambon A, Mazzone A, Cherubini A, Mossello E, et al. Delirium, Dementia, and In-Hospital Mortality: The Results From the Italian Delirium Day 2016, A National Multicenter Study. J Gerontol A Biol Sci Med Sci. 2019;74(6):910-6.

16. Francis J, Kapoor WN. Prognosis after hospital discharge of older medical patients with delirium. J Am Geriatric Soc. 1992;40:601-6.
17. O'Keeffe S, Lavan J. The Prognostic Significance of Delirium in Older Hospital Patients. J Am Geriatr Soc. 1997;45:174-8.

18. Matsuda Y, Maeda I, Morita T, Yamauchi T, Sakashita A, Watanabe $\mathrm{H}$, et al. Reversibility of delirium in Ill-hospitalized cancer patients: Does underlying etiology matter? Cancer Med. 2019. [Epub ahead of print].

19. Skelton L, Guo P. Evaluating the effects of the pharmacological and nonpharmacological interventions to manage delirium symptoms in palliative care patients: systematic review. Curr Opin Support Palliat Care. 2019;13:384-91.

20. Caraceni A, Nanni O, Maltoni M, Piva L, Indelli M, Arnoldi E, et al. Impact of delirium on the short term prognosis of advanced cancer patients. Italian Multicenter Study Group on Palliative Care. Cancer. 2000;89:1145-9.

21. Lawlor PG, Rutkowski NA, MacDonald AR, Ansari MT, Sikora L, Momoli F, et al. A Scoping Review to Map Empirical Evidence Regarding Key Domains and Questions in the Clinical Pathway of Delirium in Palliative Care. J Pain Symptom Manage. 2019;57(3):661-81.

22. Seiler A, Schubert M, Hertler C, Schettle M, Blum D, Guckenberger $M$, et al. Predisposing and precipitating risk factors for delirium in palliative care patients. Palliat Support Care. 2019:1-10. [Epub ahead of print].

23. Mercadante S, Masedu F, Balzani I, De Giovanni D, Montanari L, Pittureri $\mathrm{C}$, et al. Prevalence of delirium in advanced cancer patients in home care and hospice and outcomes after 1 week of palliative care. Support Care Cancer. 2018;26:913-9.

24. Maltoni M, Caraceni A, Brunelli, C, Broeckaert, B, Christakis, N, Eychmueller, S, et al. Prognostic factors in advanced cancer patients: evidence-based clinical recommendations-a study by the Steering Committee of the European Association for Palliative Care. J Clin Oncol. 2005;23:6240-8.

25. Gagnon P, Allard P, Masse B, DeSerres M. Delirium in Terminal Cancer: A Prospective Study Using Daily Screening, Early Diagnosis, and Continuous Monitoring. J Pain Symptom Manage. 2000;19:412-26.

26. Martín Aspas A, Benítez Macías JF, Benitez Rodriguez E, Segura Fernández E, Mogollo Galván A. Relación entre factores precipitantes y mortalidad en el delirio agitado en Cuidados Paliativos. Med Paliat. 2005;12:147-51.

27. Inouye SK. Delirium in Older Persons. N Engl J Med. 2006 Mar 16;354:1157-65.

28. Smith J, Adcok L. The recognition of delirium in hospice inpatient units. Palliat Med. 2012;26:283-5.

29. Inouye SK, Rushing JT, Foreman MD, Palmer RM, Pompei P. Does delirium contribute to poor hospital outcomes? A three site epidemiologic study. J Gen Intern Med. 1998;13:234-42.

30. Caraceni A, Simonetti F. Palliating delirium in patients with cancer. Lancet Oncol. 2009;10:164-72.

31. Centeno C, Sanz A, Vara F, Pérez P, Bruera E. Abordaje terapéutico del delirium en pacientes con cáncer avanzado. Med Paliat. 2003;10:149-56. 